\title{
Effects of golf ball dimple configuration on aerodynamics, trajectory, and acoustics
}

\author{
Chang-Hsien Tai ${ }^{+}$Chih-Yeh Chao ${ }^{++}$Jik-Chang Leong ${ }^{+}$Qing-Shan Hong ${ }^{+}$
}

Department of Vehicle engineering, National Ping-Tung University of Science and Technology ${ }^{+}$

Department of Mechanical engineering, National Ping-Tung University of Science and Technology ${ }^{++}$

\begin{abstract}
The speed of golf balls can be regarded as the fastest in all ball games. The flying distance of a golf ball is influenced not only by its material, but also by the aerodynamics of the dimple on its surface. By using Computational Fluid Dynamics method, the flow field and aerodynamics characteristics of golf balls can be studied and evaluated before the golf balls are actually manufactured. This work uses FLUENT as its solver and numerical simulations were carried out to estimate the aerodynamics parameters and noise levels for various kinds of golf balls having different dimple configurations. With the obtained aerodynamics parameters, the flying distance and trajectory for a golf ball were determined and visualized. The results showed that the lift coefficient of the golf ball increased if small dimples were added between the original dimples. When launched at small angles, golf balls with deep dimples were found to have greater lift effects than drag effects. Therefore, the golf balls would fly further. As far as noise generation was concerned, deep dimples produced lower noise levels.
\end{abstract}

Keywords: golf ball, CFD, dimple, flying trajectory, acoustics

\section{Introduction}

Many reports about golf ball, including those describe the history of its development, have introduced the standards on golf ball specification. However, there is not a single well-documented solid publication found paying attention to the requirements for the design of golf ball surface. Not only have a lot of reports discussed the material and structure of a golf ball, but also most of the golf ball manufacturers improve their products by modifying the number of layers beneath the golf ball surface and their materials. Even so, there are relatively very few papers focusing on the influence of different concave surface configurations on the aerodynamic characteristics of the golf ball. Furthermore, the noise a golf ball generates in a tournament is very likely to affect the emotion and hence the performance of the golf ball player. For these reasons, this study investigates the performance of a golf ball based on the CFD method with experimental validation by the means of a wind tunnel.

To conform to the technology progress, USGA has modified the standard requirements for golf ball [1], including the permission to use asymmetric dimple on the golf ball surface to 
make golf tournaments more interesting to watch. In 1938, Goldstein [2] had proposed an important parameter - the spin ratio. In corporation with different Reynolds numbers, this parameter makes the study of lift and drag effects feasible for whirling smooth bodies. Schouveiler, et al. [3] utilized numerical method to simulate the relationship of wake effect behind two spheres. The objective of their paper was to determine the critical Reynolds number and the interval distance between the two spheres. Jearl [4] pointed out that golf ball surface produces a thin boundary layer as it flies. Under the conventional perception, people thought that the friction force of a smooth sphere was always smaller than that of a sphere with dimples, and therefore the smooth sphere was expected to fly further. In fact, the phenomenon is exactly the opposite. Jearl showed that the flying distance of the ball with dimples was four times greater than the smooth ball because of form drag. In his book, Jorgensen [5] emphasized that the main objective of concaved surfaces on a golf ball is to generate small scale turbulence. When flying, this turbulence postpones air separation, reduces the low pressure region trailing the golf ball, and therefore lowers the air drag. Warring [6] performed a series of numerical studies related to golf balls using Excel spreadsheets. His paper included the introduction of theoretical phenomenon, the influence of drag force on the flying performance of golf balls, the estimation of Magnus force, and the prediction of golf ball trajectory. The goal of his paper was to provide guidance for golf ball players and manufacturers so that their golf ball was capable of flying for a longer distance. Eilek [7] further discussed the lift force generated by Magnus effect in his writing according to the Bemoulli's Theorem.
In the study of acoustics, Singer, et al. [8] calculated the noise level from a source using a hybrid grid system with the help of Lighthill's acoustics analytic approach. On the other hand, Montavon, et al. [9] combined CFD method and Computational Aeroacoustics Approach (CAA) to simulate noise generation from a cylinder. Using CFX-5 with LES (Large Eddy Simulation) as their turbulence model and Ffowcs-Williams Hawkings formulation, they had successfully shown that their predicted sound levels agreed very well with theoretical ones for Reynolds numbers about 1.4x $10^{5}$. However, for lower Reynolds numbers, their estimated sound pressures were $10 \mathrm{~dB}$ greater than the theoretical ones.

\section{Mathematical Model}

\subsection{Governing equations}

The present numerical simulation of the airflow distribution around a golf ball requires the use of various theoretical mathematical models based on fluid dynamics principles. The present model consists of the continuity equation, the momentum equation, and the energy equation. These equations employed in the present numerical model are presented below.

(i) Continuity equation:

$$
\frac{\partial \rho}{\partial t}+\nabla \cdot(\rho \vec{U})=0
$$

(ii) Momentum equation:

$$
\begin{aligned}
& \frac{\partial(\rho \vec{U})}{\partial t}+\nabla \bullet(\rho \vec{U}) \vec{U}=-\nabla P+\nabla \bullet\left(\mu_{v} \nabla \vec{U}\right)+\rho \vec{F} \\
& \text { where } \mu_{v}=\mu+\mu_{t}
\end{aligned}
$$

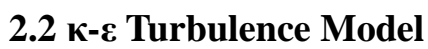

The $\kappa-\varepsilon$ turbulent model is usually applied to 
simulate the air flow field in mechanical ventilation system and also modern engineering applications. In early research, turbulent model was applied in high Reynolds number incompressible flows. But it was later experimentally proven that the air flow near the wall is associated with low Reynolds numbers. Therefore, the development of turbulence model for low Reynolds numbers has been an intensive focus for research activities. One remedy to this scenario is to introduce a wall function so that the low Reynolds number air flow near the wall and the high Reynolds number flow far away from the wall can be simulated at the same time. In this paper, the turbulent model used is the amended standard $\kappa-\varepsilon$ model because it has been proven to give good predictions for complex flows. The amended coefficient of standard $\kappa-\varepsilon$ model are $\mathrm{C}_{\mathrm{u}}=$ $0.09, \sigma_{\varepsilon}=1.30, \sigma_{\mathrm{K}}=1.00, \mathrm{C}_{1 \varepsilon}=1.44, \mathrm{C}_{2 \varepsilon}=1.92, \mathrm{C}_{3}$ $=0.8$.

The amended standard $\kappa-\varepsilon$ model is given as

$$
\begin{aligned}
& \frac{\partial(\rho k)}{\partial t}+\nabla \bullet(\rho k \mathrm{U})= \\
& \nabla \bullet\left[\frac{\mu_{t}}{\sigma_{k}} \nabla k\right]+G+B-\rho \varepsilon \\
& \frac{\partial(\rho \varepsilon)}{\partial t}+\nabla \bullet(\rho \varepsilon \mathrm{U})=\nabla \bullet\left[\frac{\mu_{t}}{\sigma_{\varepsilon}} \nabla \varepsilon\right]+ \\
& C_{1 \varepsilon} \frac{\varepsilon}{k}(G+B)\left(1+C_{3} R_{f}\right)-C_{2 \varepsilon} \rho \frac{\varepsilon^{2}}{k} \\
& G=2 \mu_{t} E_{i j} \bullet E_{i j} \\
& B=\beta g_{i} \frac{\mu}{\sigma_{T}} \frac{\partial \rho}{\partial T} \\
& \beta=-\frac{1}{\rho} \frac{\partial \rho}{\partial T} \\
& \mu_{t}=\rho C_{\mu} \frac{k^{2}}{\varepsilon} \\
& R_{f}=\frac{-G_{l}}{2(B+G)}, G_{l}=2 B
\end{aligned}
$$

\subsection{Acoustic Analogy Approach}

The sound spectra at the acoustics receivers associated with a golf ball were also calculated using the fowcs Williams - Hawkins acoustic analogy (FW-H) recently implemented in Fluent 6.1. Kim et al. [10] described the implementation of this analogy in details. It pointed out that the fowcs Williams-Hawkins acoustic analogy must satisfy the following hypothesis :

- Flows is low speed

- The contribution of the viscous and turbulent stresses are negligible in comparison with the pressure effect on the body

- The observer is located outside of the source region (i.e. Outside boundary layers is separated from flow or wakes)

The FW-H equation can be written as:

$$
\begin{gathered}
\frac{1}{a_{0}^{2}} \frac{\partial^{2} p^{\prime}}{\partial t^{2}}-\nabla^{2} p^{\prime}=\frac{\partial^{2}}{\partial x_{i} \partial x_{j}}\left\{T_{i j} H(f)\right\} \\
-\frac{\partial}{\partial x_{i}}\left\{\left[P_{i j} n_{j}+\rho u_{i}\left(u_{n}-v_{n}\right)\right] \delta(f)\right\} \\
+\frac{\partial}{\partial t}\left\{\left[\rho_{0} v_{n}+\rho\left(u_{n}-v_{n}\right)\right] \delta(f)\right\}
\end{gathered}
$$

where

$u_{i}=$ fluid velocity component in the $x_{i}$ direction $u_{n}=$ fluid velocity component normal to the surface $f=0$

$v_{i}=$ surface velocity components in the $x_{i}$ direction $v_{n}=$ surface velocity component normal to the surface $f=0$

$\delta(f)=$ Dirac delta function

$H(f)=$ Heaviside function 
$T_{i j}$ is the Lighthill stress tensor, defined as

$$
T_{i j}=\rho u_{i} u_{j}+p_{i j}-a_{0}^{2}\left(\rho-\rho_{0}\right) \delta_{i j}
$$

$P_{i j}$ is the compressive stress tensor. For a Stokesian

fluid, this is expressed as

$$
P_{i j}=p \delta_{i j}-\mu\left[\frac{\partial u_{i}}{\partial x_{j}}+\frac{\partial u_{j}}{\partial x_{i}}-\frac{2}{3} \frac{\partial u_{k}}{\partial x_{k}} \delta_{i j}\right]
$$

\section{Characteristics of geometry, grids and flow field}

The objectives of this investigation are to determine the shape of golf ball which produces different aerodynamics characteristics and then to use those shape parameters for the simulation of golf ball flying trajectory. In addition to, discuss thorough of flow field character and physical property, included the relationship between sound frequency with sphere shape. Figs. 1 and 2 show the geometry and boundary of a typical golf ball. Its surface consists of hundreds of dimples of different sizes and depths. The combination of these dimples has made the process of grid generation greatly complicated and therefore very time consuming. It is possible in some cases that two dimples may interlock with each other and eventually lead to lethal grid generation errors. Hence, this step requires extreme carefulness and the experience gained from numerous trials. 3-D grid systems contain structured grid and non-structured grid. Figs. 3 and 4 show these kinds of grid near the sphere. In those cases used non-uniform distribute grid system which could increase more mesh in key-position, this way would simulation more complete flow field near the sphere. The 3-D golf ball simulation in this paper uses structured and unstructured grid for comparison. Table 1 lists the parameters of every case. The golf ball diameter is $42.6 \mathrm{~mm}$ while the domain size is $600 \mathrm{~mm} \times 400 \mathrm{~mm} \times 400 \mathrm{~mm}$ in the $\mathrm{x}, \mathrm{y}$, and $\mathrm{z}$-directions.

\section{Validation}

For validation, this study used a 3-D sphere. The turbulence model being validated is the standard $\kappa-\varepsilon$ model. The drag coefficient of the sphere starts to drop off at a Reynolds number of $2 \times 10^{5}$. This corresponds to the transition of air flow from laminar to turbulent. Drag coefficient is the lowest at the critical Reynolds number of $4 \times 10^{5}$. After that, drag coefficient will raise slowly with Reynolds number. Figures 5 show the comparison of drag coefficients at different Reynolds number Schlichting [11] provided (in Fig. 5(a)) and those obtained through this study (in Fig. 5(b)). These results qualitatively agree well with each other. Although the values of critical Reynolds number are not exactly the same, the computational prediction is acceptable as far as the overall trend is concerned.

This study uses a 2-D cylinder to validate the noise simulation. The flow was set as air, the outside pressure was set 1 atm., the inlet velocity was $69.19 \mathrm{~m} / \mathrm{s}\left(\operatorname{Re}=5 \times 10^{5}\right)$, and pressure outlet was applied at the outlet boundary. Both DES and LES turbulence models were applied to simulate the sound field. Figure 6 showed the result of numerical simulations for comparison. The spectrum analysis performed through LES AA 
model is almost the same with that through CAA model [12]. However, the result of DES turbulence model is similar to the other two cases if the frequency is less than $3000 \mathrm{~Hz}$. At higher frequencies, the discrepancy is large. It is believed that this is attributed to the fact that the accuracy of DES model is on the first order whereas that of LES model is on the second order.

\section{Result and discuss}

This study used structured and non-structured grids for numerical simulation. Figure 7 showed the drag coefficients of two types of grid. Since the benchmark values for drag coefficient are between $0.25 \sim 0.27$ [13], the drag coefficient obtained is closer to the benchmark values via structured grid simulation than non-structured grid. On the other hand, according to the performance test by the manufacturer of this golf ball, the actual flying distance of this ball was $240 \mathrm{~m}$. Figure 8 showed the flying distance which was $268.1 \mathrm{~m}$ obtained from the simulation using non-structured grids. It had an error of $11.7 \%$ compared with the actual distance. The distance predicted by the structured grid simulation was $225.2 \mathrm{~m}$, which had an error of $6.2 \%$. Judging based on flying distance, a simulation based on a structured grid system produces a higher accuracy. However, both the structured and non-structured grid systems are qualitatively reliable for the trends of drag coefficient obtained through both these systems produce are the same.

The speed of the golf ball considered in this study ranges from $0.345 \mathrm{~m} / \mathrm{s}$ to $83.82 \mathrm{~m} / \mathrm{s}$. This corresponds to Reynolds numbers ranging from $1 \times$ $10^{3}$ to $2.43 \times 10^{5}$. Figure 9 shows the flow field around a typical golf ball (Case 1). In Case 2, additional dimples are added onto the original golf ball surface considered in Case 1. The orientation of these additional dimples is depicted in Figure 10. It is found, based on Figure 10, that the flow field associated to Case 2 is no longer symmetrical because of the presence of the additional dimples. Figure 11 demonstrates the distribution of lift and drag coefficients of Cases 1 and 2. Clearly, the addition of small dimples increases the drag. Especially when the Reynolds number is small, the increase in drag is greater. For greater Reynolds numbers, the increase in drag is almost consistent. This implies that the golf ball in Case 2 suffers more serious drag effect at low trajectory speeds. Also shown in the figure, the lift the golf ball in Case 2 experiences at moderate Reynolds numbers increases so greatly that it becomes greater than that for Case 1. The life force in overall is therefore greater for Case 2 than Case 1. The results of these two cases are compared and shown in Figure 12 in terms of golf ball flying trajectory. Although the drag imposed on the golf ball is always smaller for Case 1 than for Case 2, the drag in Case 1 is only about $38.5 \%$ less than that in Case 2. However, the lift in Case 2 is $103 \%$ greater than that in Case 1. This somewhat indicates the lift effect is 2.68 times of the drag effect. The overall performance of the golf ball for Case 2 is much greater than that for Case 1. Therefore, the golf ball for Case 2 is capable of traveling further, as shown in Figure 12.

Cases $3 \sim 7$ investigated the effect of five different dimple depths on the golf ball flying performance under the condition that the golf ball coverage areas are the same. Table 1 lists the details of these five cases. Figures 13 and 14 show the drag and lift of these cases, respectively. In 
these figures, it is obvious that drag coefficient increases with dimple depth. As far as the lift coefficient is concerned, they increase with dimple depth for Cases $3 \sim 5$, but decreases for Cases 6 and 7. If swung at large launch angles, the golf ball would stay in the sky for a longer duration and therefore its drag effect is greater than its lift effect. This leads to the fact that the flying distance is inversely proportional to the dimple depth (Figure 15). In contrast, if swung at low launch angles, the duration the golf ball would stay in the sky is considerably shorter. In this case, its lift effect becomes greater than its drag effect and thus the flying distance is directly proportional to the dimple depth (Figure 16). Even so, the flying distance associated to a low launch angle is found to behave in the reverse manner when the dimple depth exceeds $0.25 \mathrm{~mm}$. Generally, the range of a golf ball launch angle between $10^{\circ} \sim 12^{\circ}$ can be considered as within the low launch angle range. This study suggests that the design of golf balls with deep dimple can the lift of the golf balls and improve their flying distance as long as the dimple depth is less than $0.25 \mathrm{~mm}$.

In the prediction of noise, Table 2 lists the position of noise detectors. This section only considered Cases $3 \sim 5$ by setting the body of the golf ball to be the sound source to examine the different noise level produced in conjunction with different dimple depths. The reference sound pressure employed in this paper is the international standard sound pressure (20 $\mu \mathrm{pa})$. Most noises were produced as a result of eddy motion. Figure 17 showed the magnitude of the vorticity due to eddy production by the dimples when air flowed pass the golf ball surface in Case 5. The maximum eddy motion took place near the center of the golf ball surface. The regions with a high vorticity intensity shown in Fig. 17 were the places where noise was generated. Figure 18 shows the spectrum analysis of these three cases whose Overall Sound Pressure levels at detector point 1 were $75.3 \mathrm{~dB}, 71.9 \mathrm{~dB}$, and $58.8 \mathrm{~dB}$ for Cases $3 \sim 5$. Figure 19 shows the Overall Sound Pressure Levels for the four detectors.

\section{Conclusion}

This study has examined various conditions for the problem considered. The flying distance of the golf ball is used as the criterion to quantify the success of a simulation. Based on this study, several conclusions can be drawn as follows:

(1) As far as the selection of grid distribution is concerned, structured grid will produce more accurate results. Unfortunately, simulations with structured grid normally take longer time to accomplish. Nowadays, this can be overcome by using parallel computation technique. As a matter of fact, the results obtained from non-structured grid qualitatively resemble those from structured grid. Therefore, simulations based on non-structured grid are very useful in providing preliminary understanding of a problem.

(2) Adding small dimples to the original golf ball surface increases both the drag and lift as evidently shown in Cases 1 and 2. Between these two cases, the amount of lift force increased was 2.86 greater than drag causing lift effect to be greater than drag effect and making the sphere of Case 2 fly farther.

(3) With the same coverage area, it is found that the golf ball with deeper dimples is associated to greater drag and lift. Hence, the flying distance of a specific golf ball design should 
be examined with a given swing launch angle. When launched at large angles, the flying distance of the golf balls with deep dimples are short whereas, when launched at small angles, the flight distance of golf balls with deep dimples are longer. Furthermore, the threshold depth of a golf ball is about $0.25 \mathrm{~mm}$.

(4) In our analysis of noise, we have considered three cases whose dimple depth is less than the threshold value (Cases $3 \sim 5$ ) to examine the relationship between the depth of dimple with noise. By judging the noise value based on the Overall Sound Pressure Level, the noise value of Case 3 was the highest and Case 5 was the lowest. This means that golf balls with deep dimples produced the least noise.

\section{Acknowledgement}

The authors gratefully acknowledge SCANNA CO., LTD for their generous support of this work.

\section{Reference}

[1] Gelberg, J. N., "The Rise and Fall of the Polara Asymmetric Golf Ball: No Hook, No Slice, No Dice," Technology In Society, Vol. 18, No. 1, pp. 93-110, 1996.

[2] Goldstein, S., "Modern Developments in Fluid Dynamics," Vols. I and II. Oxford: Clarendon Press, 1938.

[3] Schouveiler, L., Brydon, A., Leweke, T. and Thompson, M. C., "Interactions of the wakes of two spheres placed side by side," Conference on Bluff Body Wakes and Vortex-Induced Vibrations, pp. 17-20, 2002.

[4] Jearl, W., "More on boomerangs, including their connection with the dimpled golf ball," Scientific American, pp. 180, 1979.

[5] Jorgensen, T. P., "The Physics of Golf, 2nd edition," New York: Springer-Verlag, pp. 71-72, 1999.

[6] Warring, K. E., "The Aerodynamics of
Golf Ball Flight," St. Mary's College of Maryland, pp. 1-37, 2003.

[7] Eilek, J. A., "Vorticity," Physics 526 notes, pp. 38-46, 2005.

[8] Singer, B. A., Lockard, D. P. and Lilley, G. M., "Hybrid Acoustic Predictions," Computers and Mathematics with Application 46, pp. 647-669, 2003.

[9] Montavon, C., Jones, I. P., Szepessy, S., Henriksson, R., el-Hachemi, Z., Dequand, S., Piccirillo, M., Tournour, M. and Tremblay, F., "Noise propagation from a cylinder in a cross flow: comparison of SPL from measurements and from a CAA method based on a generalized acoustic analogy," IMA Conference on Computational Aeroacoustics, pp. 1-14, 2002.

[10] Kim, S.E., Dai, Y., Koutsavdis, E.K., Sovani, S.D., Kadam, N.A., and Ravuri, K.M.R, "A versatile implementation of acoustic analogy based noise prediction approach," AIAA 2003-3202, (2003)

[11] Schlichting, H., "Boundary-Layer Theory, 7th ed.," New York: McGraw-Hill, 1979.

[12] Fluent Inc., "Aero-Noise Prediction of Flow Across a Circular Cylinder," Fluent 6.1 Tutorial Guide, 2002.

[13] Mehta, R. D., 1985, "Aerodynamics of Sports Balls," in Annual Review of Fluid Mechanics, ed. by M. van Dyke, et al. Palo Alto, CA: Annual Reviews, pp. 151-189.

Table 1 Parameter illustrate of cases

\begin{tabular}{cc}
\hline Case & Variable of sphere \\
\hline Case 1 & Depth of dimple is $0.178 \mathrm{~mm}$ \\
Case 2 & Case 1+small dimple \\
\hline Case 3 & Depth of dimple is $0.15 \mathrm{~mm}$ \\
Case 4 & Depth of dimple is $0.2 \mathrm{~mm}$ \\
Case 5 & Depth of dimple is $0.25 \mathrm{~mm}$ \\
Case 6 & Depth of dimple is $0.3 \mathrm{~mm}$ \\
Case 7 & Depth of dimple is $0.35 \mathrm{~mm}$ \\
\hline
\end{tabular}

Table 2 coordinates of acoustics receiver locations

\begin{tabular}{cccc}
\hline point & $\mathrm{x}(\mathrm{m})$ & $\mathrm{y}(\mathrm{m})$ & $\mathrm{z}(\mathrm{m})$ \\
\hline 1 & -0.4 & 0.05 & 0.05 \\
2 & -0.4 & -0.05 & 0.05 \\
3 & -0.4 & 0.05 & -0.05 \\
4 & -0.4 & -0.05 & -0.05 \\
\hline
\end{tabular}




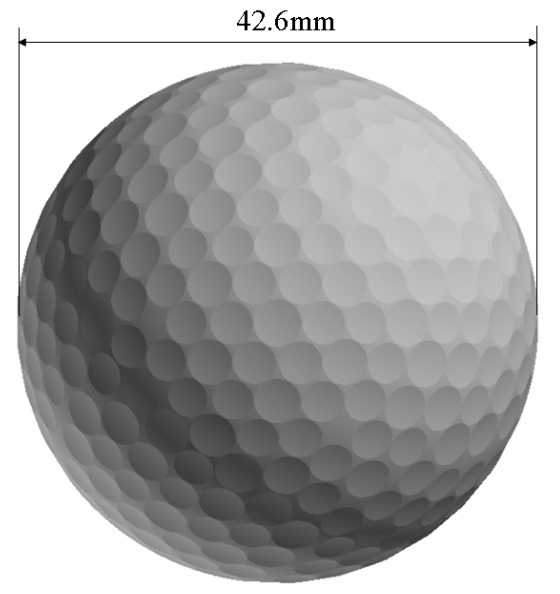

Figure 1 Geometric and size of golf ball

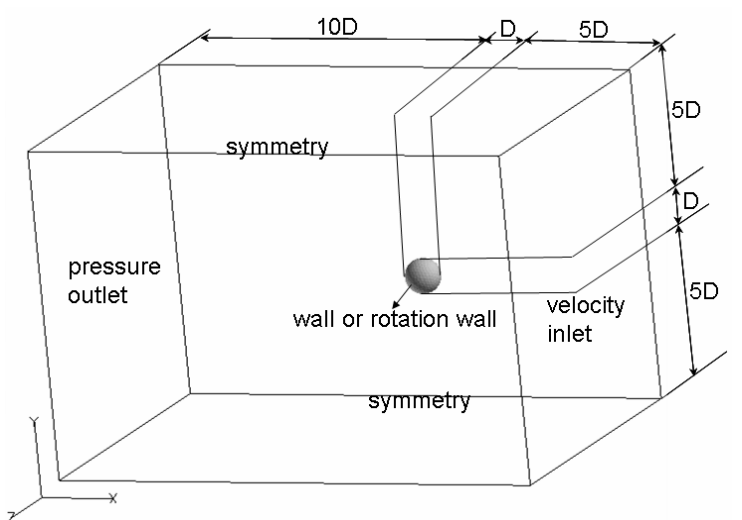

Figure 2 Boundary condition and domain size

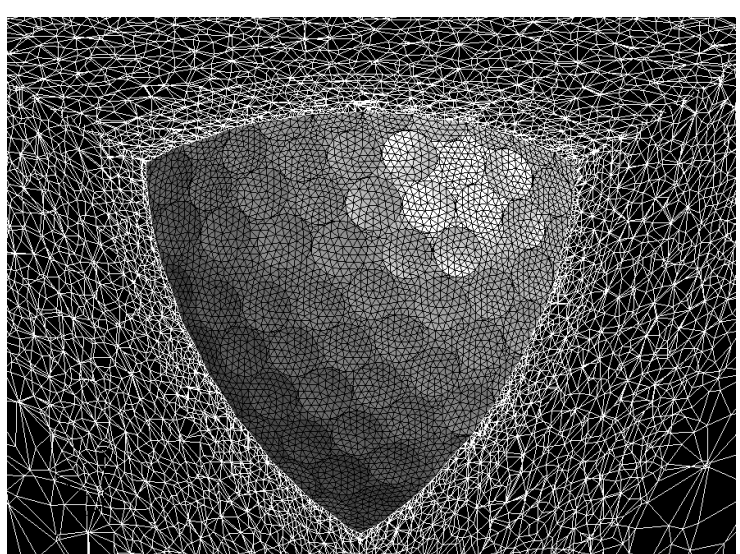

Figure 3 Non-structured grid near the sphere

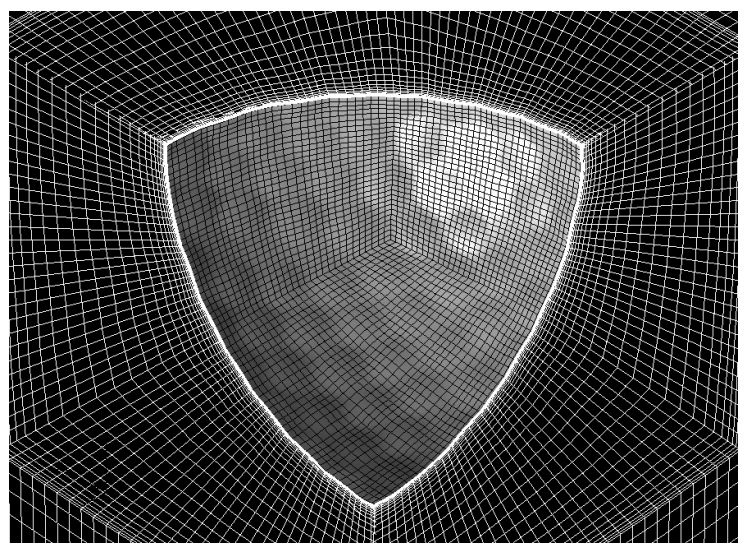

Figure 4 Structured grid near the sphere

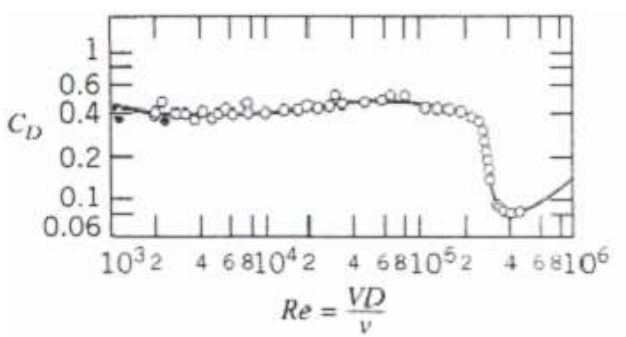

(a)

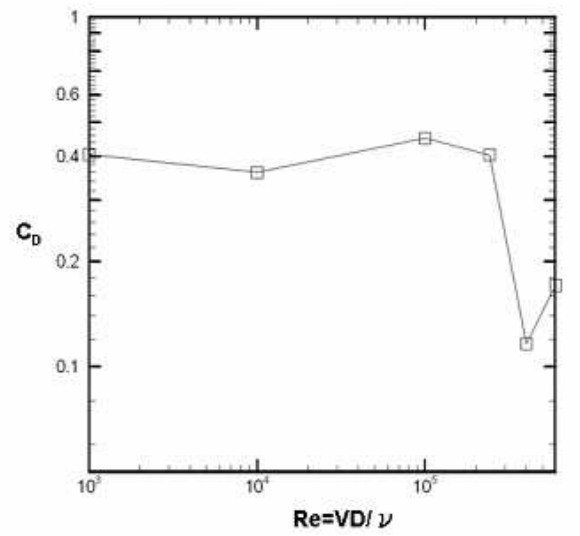

(b)

Figure 5 Validation of the sphere: (a) experiment value of Schlichting [11], (b)this study proof

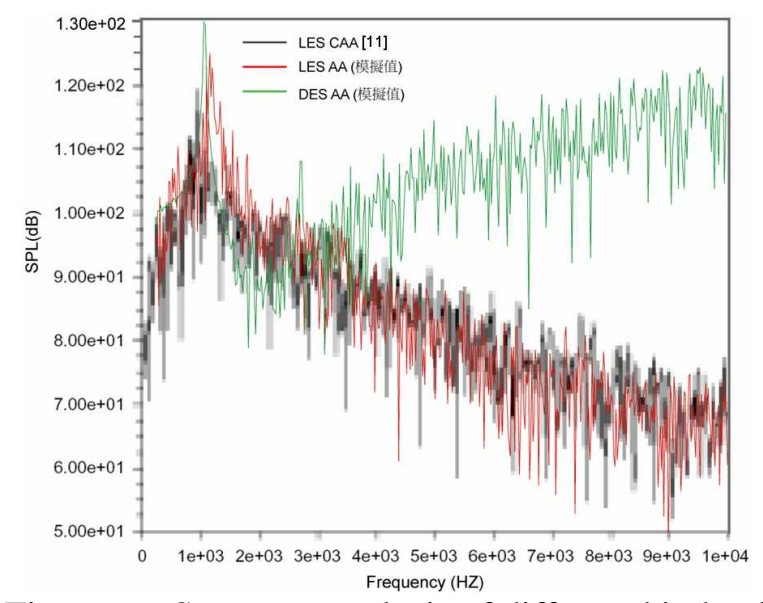

Figure 6 Spectrum analysis of different kinds of turbulence model $\left(\mathrm{Re}=5 \times 10^{5}\right)$

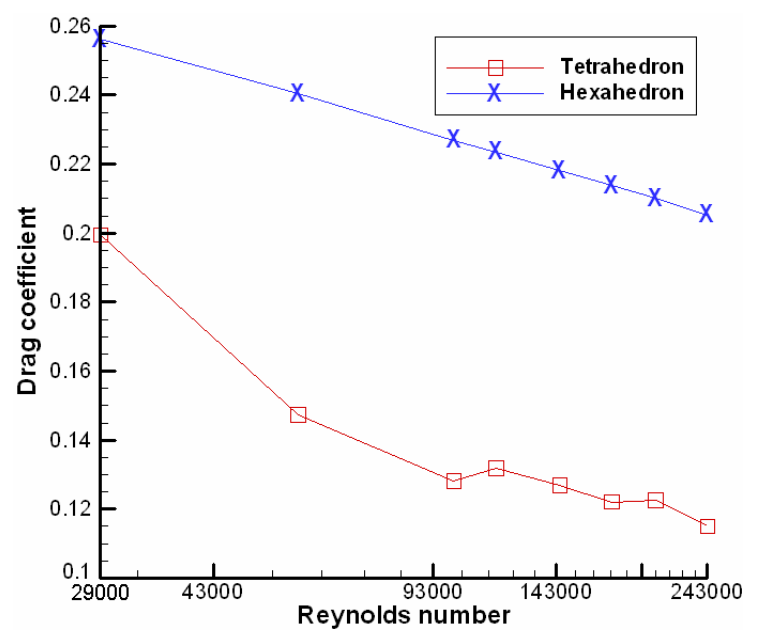


Figure 7 Drag coefficients for structured and non-structured grid systems

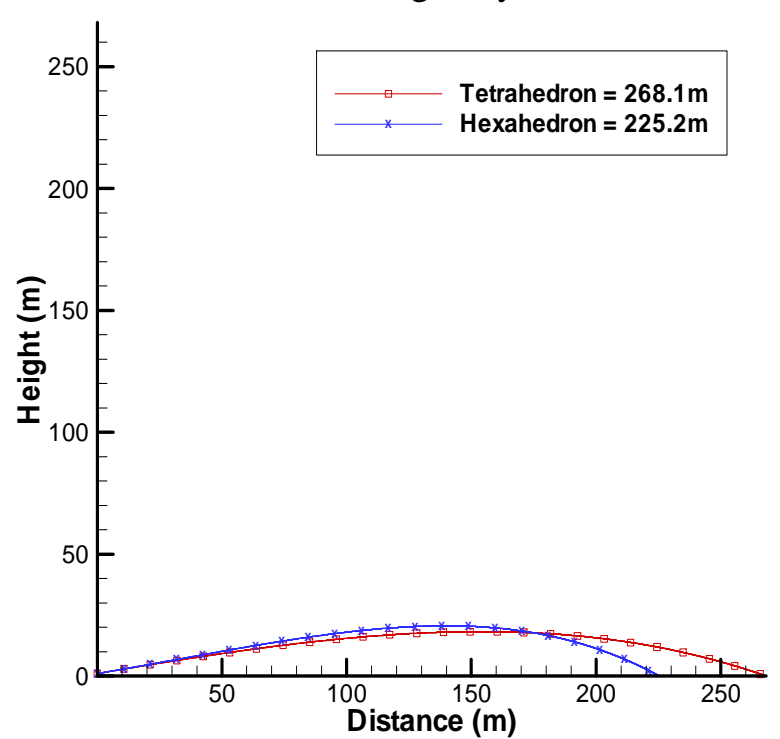

Figure 8 Golf ball flying trajectories for structured and non-structured grid systems



Figure 9 Velocity vector for Case 1 with spinning $\left(\mathrm{Re}=1 \times 10^{5}\right)$

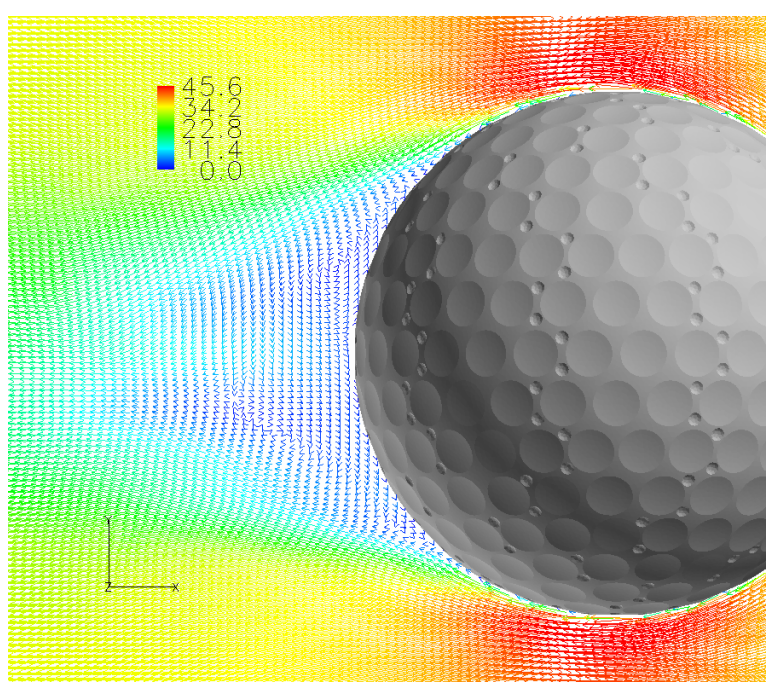

Figure 10 Velocity vector for Case $2\left(\operatorname{Re}=1 \times 10^{5}\right)$

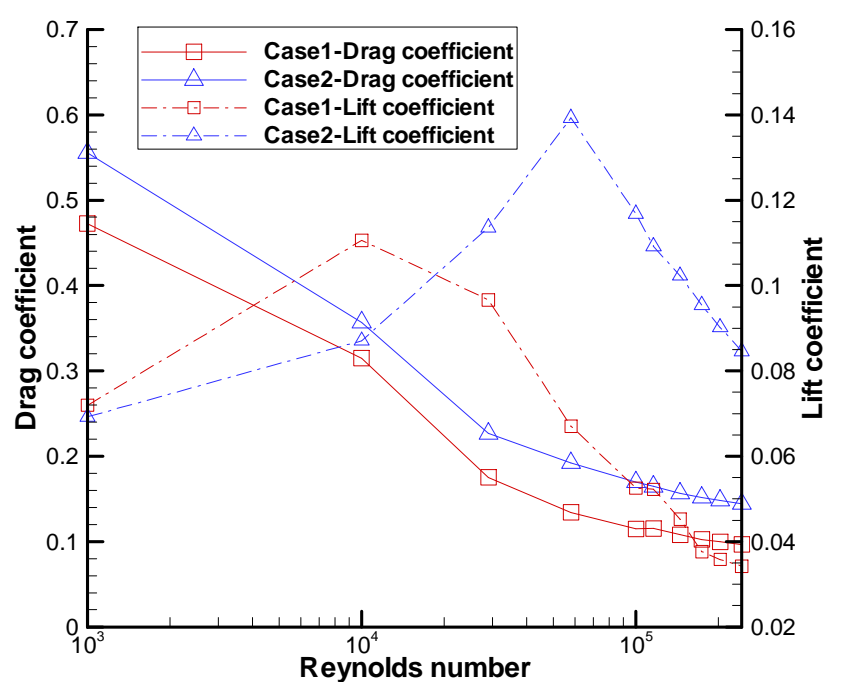

Figure 11 Drag and Lift coefficients for Cases 1 and 2

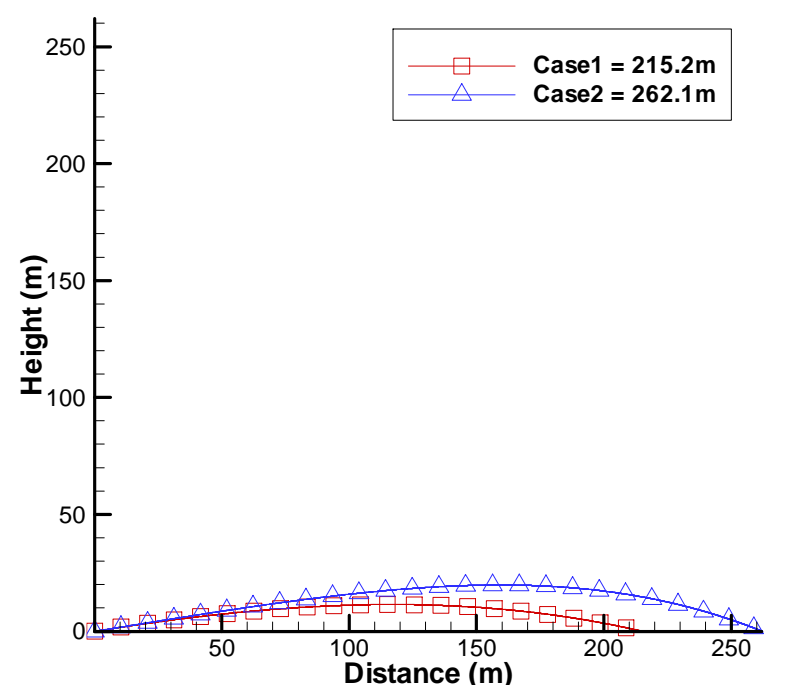

Figure 12 Flying trajectory of Case 1 and Case 2 


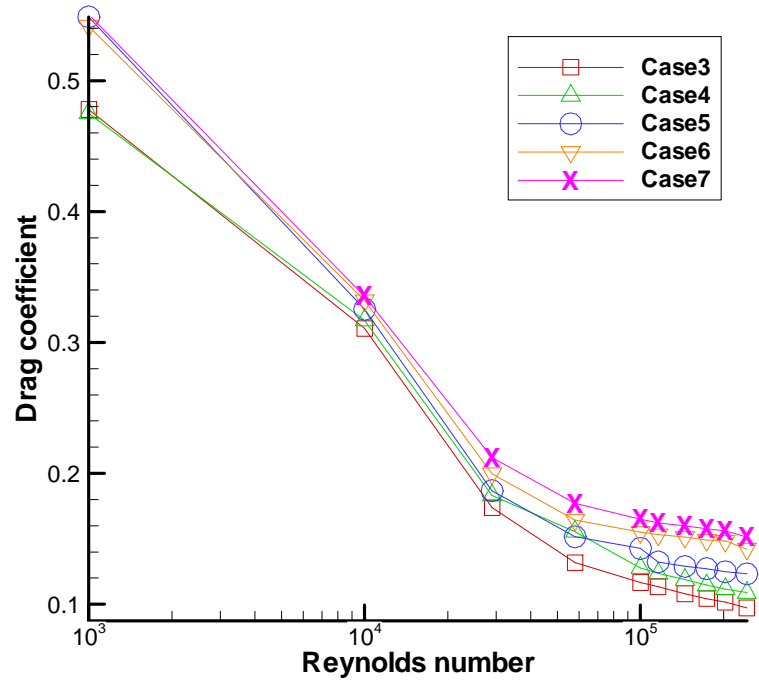

Figure 13 Drag coefficients for Cases $3 \sim 7$

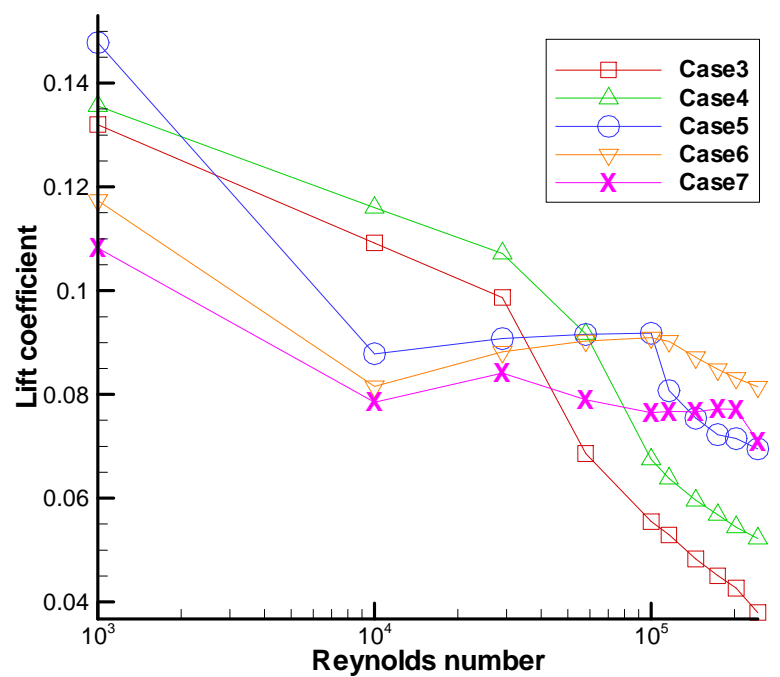

Figure 14 Lift coefficients for Cases $3 \sim 7$

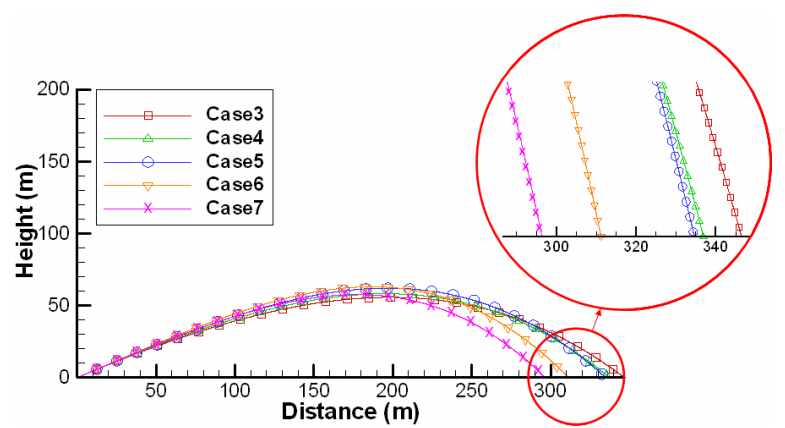

Figure 15 Flying trajectories for Cases $3 \sim 7$ ( $25^{\circ}$ launch angle)

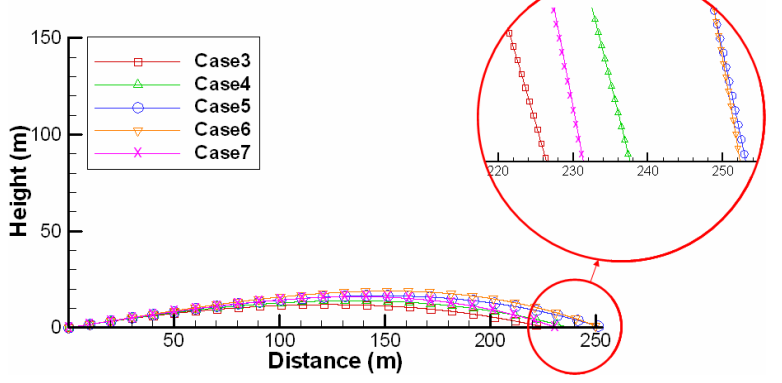

Figure 16 Flying trajectories for Cases $3 \sim 7$ $\left(10^{\circ}\right.$ launch angle)

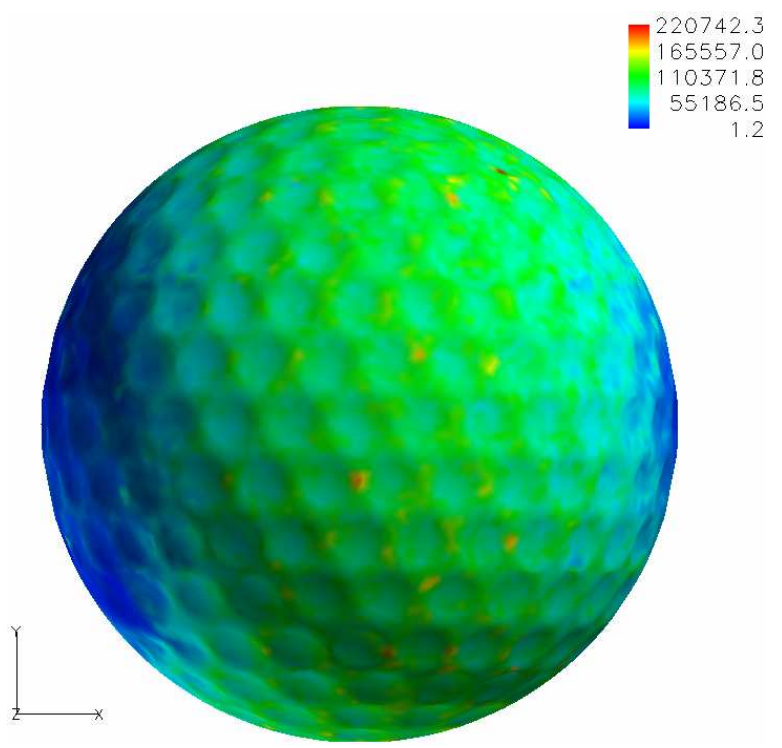

Figure 17 Vorticity magnitude for Case 5 (unit:1/s)

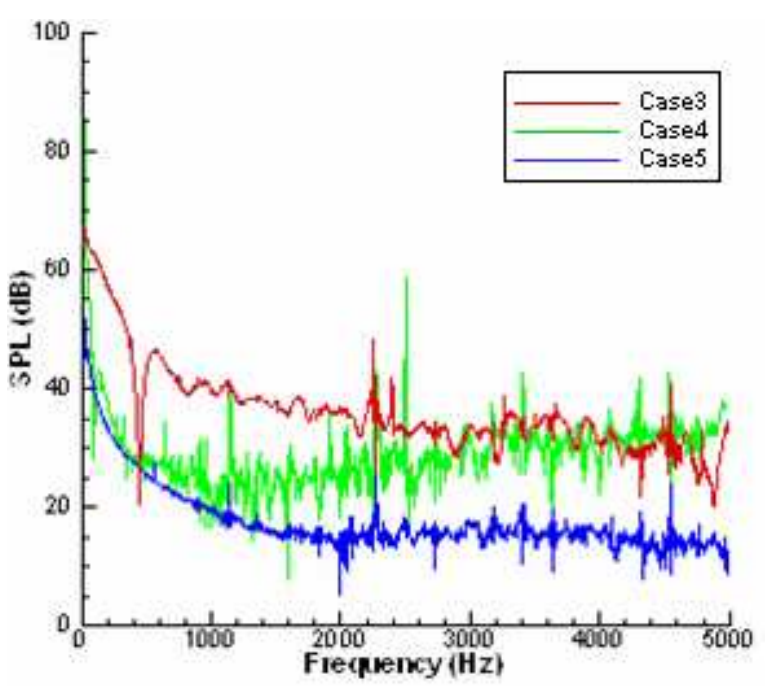

Figure 18 Noise spectrums for Cases $3 \sim 5$ (point1) 


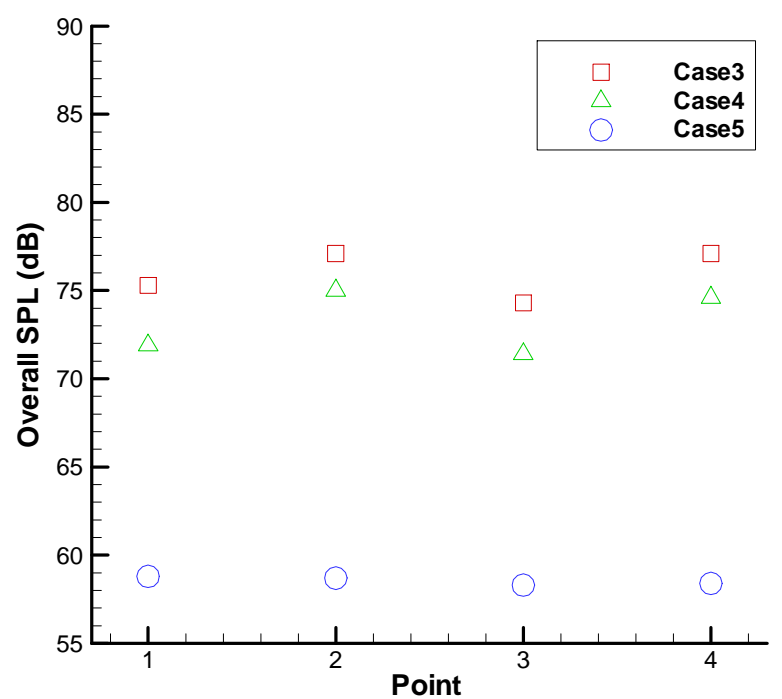

Figure 19 Noise levels at each detector for Case $3 \sim$ Case 5 